\title{
FAIVRE (Antoine), HANEGRAAFF (Wouter J.), éds., Western Esotericism and the Science ofReligion
}

Louvain, Peeters, 1998, XVII+309 p. (illustr., index) (coll. « Gnostica », $\mathrm{n}^{\circ} 2$ )

\section{Marco Pasi}

\section{OpenEdition}

\section{Journals}

Édition électronique

URL : http://journals.openedition.org/assr/20543

DOI : $10.4000 /$ assr. 20543

ISSN : $1777-5825$

Éditeur

Éditions de l'EHESS

\section{Édition imprimée}

Date de publication : 1 juillet 2000

Pagination : 71-74

ISBN : 2-222-96691-4

ISSN : 0335-5985

Référence électronique

Marco Pasi, «FAIVRE (Antoine), HANEGRAAFF (Wouter J.), éds., Western Esotericism and the Science ofReligion ", Archives de sciences sociales des religions [En ligne], 110 | avril-juin 2000, document

110-17, mis en ligne le 19 août 2009, consulté le 21 décembre 2020. URL : http://

journals.openedition.org/assr/20543; DOI : https://doi.org/10.4000/assr.20543

Ce document a été généré automatiquement le 21 décembre 2020.

(c) Archives de sciences sociales des religions 


\title{
FAIVRE (Antoine), HANEGRAAFF (Wouter J.), éds., Western Esotericism and the Science ofReligion
}

Louvain, Peeters, 1998, XVII+309 p. (illustr., index) (coll. « Gnostica », $\left.\mathrm{n}^{\circ} 2\right)$

\author{
Marco Pasi
}

\section{RÉFÉRENCE}

FAIVRE (Antoine), HANEGRAAFF (Wouter J.), éds., Western Esotericism and the Science ofReligion,Louvain, Peeters, 1998, XVII+309 p. (illustr., index) (coll. « Gnostica », n²)

Une nouvelle discipline académique est en train de naître. Il s'agit de l'étude de l'ésotérisme occidental. C'est ce que nous annonce W.J.H. dans son introduction à ce choix de contributions présentées au dernier colloque de l'International Association for the History of Religion (Mexico, 1995), lors d'une session spécifique portant le même titre que le recueil. En réalité la discipline devrait être considérée pour le moins adolescente, la première chaire universitaire à être consacrée à l'étude de l'ésotérisme étant celle créée en 1965 au sein de l'École Pratique des Hautes Études, intitulée « Histoire de l'ésotérisme chrétien » et occupée jusqu'en 1979 par François Secret. Cette année-là la chaire passa, avec une modification de son intitulé (devenu « Histoire des courants ésotériques et mystiques dans l'Europe moderne et contemporaine »), à A.F, qui en est encore le titulaire. Pourquoi alors parler aujourd'hui de la " naissance d'une discipline»? Effectivement ces dernières années quelque chose de nouveau s'est produit dans le domaine en question, en particulier sous deux aspects. Premièrement, s'il est vrai qu'il existait déjà une longue tradition d'études sérieuses, en français comme en d'autres langues, dont le sujet pouvait conduire au champ des courants ésotériques, il n'y avait pas, par contre, une réflexion qui portât sur le domaine dans son ensemble, sur sa spécificité et son homogénéité. De plus, les travaux d'A.F., étaient 
surtout de type historique et philologique, consacrés à des auteurs ou à des aspects ponctuels. Il manquait une réflexion méthodologique et épistémologique sur l'étude de l'ésotérisme en général et son statut à l'intérieur du champ plus vaste des sciences religieuses.

En deuxième lieu, une seule chaire ne fait pas une discipline, mais plutôt une idée de discipline. Pour que cette discipline se concrétise, ou plutôt - comme le dit W.J.H. dans son introduction -, se "normalise ", il faut que d'autres chaires soient créées dans le monde, ainsi que des réseaux de chercheurs, des revues, des colloques, des collections de livres. En ce qui concerne le premier aspect, depuis la première moitié des années 1990, des réflexions sur la spécificité de l'étude de l'ésotérisme ont commencé à voir le jour, particulièrement en France, grâce surtout aux travaux d'A.F., Jean-Pierre Laurant et Pierre Riffard (tous les trois présents dans ce recueil avec une contribution). Le travail de systématisation d'A.F. s'est révélé une source d'inspiration très féconde et a stimulé bon nombre de chercheurs, en France comme à l'étranger. À partir de la moitié des années 1990, c'est surtout W.J.H. qui a approfondi et élargi la perspective ouverte par A.F., d'abord avec son gros travail sur le Nouvel Age (New Age Religion and Western Culture, Leyde, Brill, 1996 [cf. Arch. $102 \mathrm{n}^{\circ}$ 26]) puis avec un certain nombre d'articles. En ce qui concerne le deuxième aspect quelque chose est également en train de bouger. Tout récemment, W.J.H. a été nommé professeur à l'université d'Amsterdam. Ce sera la seconde chaire au monde consacrée à l'étude des courants ésotériques. On annonce aussi des transformations et des améliorations pour la revue Aries, créée en 1985 par A.F., alors que d'autres revues, ainsi que des collections, viennent de voir le jour (par exemple la revue en ligne sur Internet Esoterica, dirigée par Arthur Versluis, et la collection de textes et d'études «Gnostica » chez Peeters, dans laquelle ce recueil a été publié). W.J.H. est d'ailleurs en train d'établir un réseau de spécialistes qui pourrait un jour se transformer en association internationale pour l'étude des courants ésotériques.

3 Ce petit rappel était peut-être nécessaire pour faire comprendre le contexte dans lequel se situe la parution de cet ouvrage (ainsi que le colloque qui en est à l'origine). Venonsen donc au contenu. Dans son introduction W.H.J. souligne l'importance que les études de Frances Yates, à partir de son livre sur Giordano Bruno de 1956, ont eue pour la reconnaissance académique de certains aspects de la culture de la Renaissance jusqu'alors négligés. Toutefois, selon W.J.H., il serait souhaitable aujourd'hui que la recherche sur l'ésotérisme aille au-delà des thèses de F. Yates et se libère de certains aspects discutables de son œuvre, qu'il appelle le « paradigme Yates». En effet certains chercheurs ont critiqué à plusieurs reprises l'accent presque exclusif que ce dernier a mis sur le réveil de la tradition " hermétique » chez certains auteurs de la Renaissance, au détriment d'autres éléments et d'autres influences (comme le néoplatonisme ou la scolastique) qui jouèrent chez ces auteurs un rôle tout aussi important. On a critiqué aussi la fameuse thèse de $\mathrm{F}$. Yates, selon laquelle la redécouverte de la tradition hermétique aurait été liée à la naissance de la nouvelle culture scientifique, qui se développa entre le XVI ${ }^{e}$ et le XVII ${ }^{e}$ siècles. W.J.H. ne semble voir dans cette thèse que la tentative de donner une certaine "légitimité » à l'étude des courants ésotériques en montrant leur importance dans l'histoire de la culture occidentale et en mettant en cause l'idée de "marginalité » qui leur est rattachée. Selon W.J.H. la recherche sur les courants ésotériques est désormais assez mûre et " légitimée », pour aller au-delà de la thèse « moderniste » et apologétique de F. Yates. 
4 Les trois premiers articles portent sur des questions de méthodologie (A.F., W.J.H. et Pierre Riffard), et semblent aborder plus directement le thème suggéré par le titre de l'ouvrage. Dans son article A.F. traite un aspect fondamental pour toute discipline, et $a$ fortiori pour une discipline nouvelle comme celle en question. Il s'agit de la terminologie et de la définition de certaines notions générales qui sont employées habituellement dans l'étude des courants ésotériques. A.F. s'occupe d'abord du terme le plus général, à savoir "ésotérisme » (en anglais "esotericism»), en expliquant dans quel sens il convient de l'utiliser dans la recherche universitaire. Il distingue ensuite les termes qui indiquent des "notions » et ceux qui indiquent des "courants", tout en tenant compte du fait que certains termes peuvent indiquer, selon les cas, soit des notions soit des courants historiques. On a donc affaire à des courants qui ne sont pas des notions (par ex. l'hermétisme ou le paracelsisme), des courants qui correspondent aussi à des notions (par ex. l'alchimie, l'astrologie et la magie), et enfin des notions qui ne sont pas des courants (par ex. la gnose). Voilà une mise au point très utile qui servira à rendre certaines discussions entre spécialistes un peu moins confuses et à réduire le risque des malentendus. Le long article de W.J.H. porte sur la «construction des 'traditions ésotériques'». W.J.H. défend une méthode «empirique » ou " historicocritique " dans l'étude des courants ésotériques, qui ne s'intéresse pas à l'idée d'un ésotérisme « en soi » et ne cherche pas à définir l'essence d'un ésotérisme « universel». L'approche empirique considère en revanche les définitions de l'ésotérisme comme des " constructions" intellectuelles nécessaires à la recherche, mais toujours relatives et non concernées par une réalité " ultime » quelconque. La tendance " universalisante " ne pourrait en revanche, selon W.J.H., que nuire au développement de la discipline. Cette tendance peut se présenter sous deux formes, opposées et spéculaires : l'une proésotérique, et l'autre anti-ésotérique. Il est impossible de suivre ici la discussion et les argumentations de W.J.H. dans les détails. Nous nous limitons ici à deux remarques. Tout d'abord, si nous partageons dans l'ensemble sa position et ses critiques, il nous semble néanmoins que dans certains cas la cible des critiques du chercheur hollandais ne soit pas très bien choisie. Par exemple, est-il légitime de prendre Éric Voegelin comme exemple d'approche universalisante anti-ésotérique? Est-on sûr que les théories de Voegelin sur la persistance de formes de pensée " gnostique » dans certains mouvements politiques contemporains soient en rapport avec les courants ésotériques tels que nous l'entendons ici ? Le point de contact semble être tout simplement l'emploi du terme "gnosticisme" par Voegelin - terme qui est parfois confondu avec, ou entendu comme synonyme de, ésotérisme. Est-ce que cela suffit pour considérer Voegelin comme un auteur pertinent pour le propos de W.J.H. ? Nous ne le croyons pas. Ensuite et indépendamment de notre remarque précédente, force est de constater que W.J.H., ici comme dans d'autres de ses études récentes, est en train d'élaborer un " paradigme » de la recherche en ésotérisme, auquel, tôt ou tard, tous les chercheurs dans le domaine finiront par devoir se confronter, quelle que soit leur opinion sur celui-ci. De ce point de vue il est intéressant de noter que l'un des auteurs présents dans le recueil, à savoir P. Riffard, est choisi, et critiqué, par W.J.H. comme un exemple parmi d'autres de l'approche universalisante pro-ésotérique. On aurait donc aimé voir dans l'article de Riffard, qui porte d'ailleurs lui aussi sur des questions de méthodologie, sinon une réponse, du moins une référence aux critiques de W.J.H., susceptible d'ouvrir une discussion sur les points mis en cause par le chercheur hollandais. Dans son article Riffard compare deux "méthodes» dans l'étude de l'ésotérisme: l'«interne» (propre aux ésotéristes), et l'«externe» (propre aux 
historiens). Il semble voir des mérites et des défauts dans les deux et finalement préfère ne pas faire son choix, ce qui justifie, de notre point de vue, les critiques de W.J.H.

Les autres articles touchent à des aspects spécifiques de l'histoire des courants ésotériques et donnent un très bon aperçu, par la variété des sujets abordés et des époques concernées, de l'état actuel de la recherche dans ce domaine. On se réjouit de voir autant d'érudition et de finesse dans ces études, d'autant plus qu'il s'agit de sujets qui jadis n'avaient leur place qu'en marge d'autres disciplines. Il est impossible de les examiner ici de façon approfondie. Nous rappelons tout simplement quelques éléments significatifs. Certains articles ouvrent des pistes nouvelles de recherche. C'est le cas de la contribution de Dan Merkur, qui porte sur l'origine d'une notion très répandue dans les théories magiques des courants occultistes, à savoir l'existence d'un "autre monde " (Otherworld), le plus souvent appelé "plan astral», qui entretient avec le monde physique une relation étroite d'analogie et d'interdépendance. Merkur voit dans un texte cabbalistique du XIII ${ }^{e}$ siècle (le Chapitre sur la Kavvanah) un point de départ possible pour l'histoire de cette notion. Italo Ronca analyse un texte alchimique arabe de l'auteur connu dans l'Occident latin comme "Senior Zadith» ( $\mathrm{X}^{\mathrm{e}}$ siècle) et l'influence qu'il a exercée dans un contexte chrétien après sa traduction en latin. L'article de Joseph Dan se concentre sur les rapports entre Cabbale juive et Cabbale chrétienne à la Renaissance, en illustrant différences et similarités qui vont se créer entre les deux courants. Ce faisant, il donne aussi un aperçu de son approche " contingentai » (contingentai) dans l'étude de l'ésotérisme. Arthur Versluis examine la présence d'un discours de type alchimique dans la littérature théosophique chrétienne, en particulier chez Johann Georg Gichtel, John Pordage, et dans certaines communautés théosophiques américaines entre le XVIII et et le XIXe siècle. Jan Snoek pose le problème préliminaire du rapport entre la franc-maçonnerie et l'ésotérisme, sans donner de réponses définitives mais en souhaitant que l'étude des courants ésotériques puisse comprendre aussi ce phénomène de la culture occidentale, qui semble avoir du mal à trouver une identité propre comme sujet de recherche universitaire. La suite de son article est consacrée à l'origine de certains grades maçonniques. Quant à Jean-Pierre Laurant, il présente un véritable exemple d'ésotérisme maçonnique avec un extraordinaire manuscrit du début du XIX ${ }^{\mathrm{e}}$ siècle, qu'il étudie depuis quelques années et auquel il a consacré plusieurs séries de conférences à l'École Pratiques des Hautes Études. L'article suivant, assez long, de Jane Williams-Hogan, est particulièrement intéressant car il contient une tentative d'application des critères introduit par A.F. pour l'identification des courants ésotériques. J. Williams-Hogan, spécialiste reconnue d'Emmanuel Swedenborg, cherche à évaluer le rapport du scientifique et visionnaire suédois avec l'ésotérisme. L'utilisation des critères d'A.E ne semble pas permettre une identification de Swedenborg comme auteur ésotérique tout court. En effet, l'un des quatre éléments essentiels, selon A.F., pour pouvoir parler d'ésotérisme stricto sensu semble être absent chez lui, à savoir la notion de nature vivante. En tout cas, que Swedenborg lui-même ait été ou non un auteur de type ésotérique, ce qui est certain, comme le montre bien l'excellente analyse de J. Williams-Hogan, est qu'il a influencé un nombre remarquable de courants et d'auteurs qui sont sans aucun doute ésotériques. Le recueil se conclut par deux études sur les conceptions de l'histoire dans certains courants, et notamment chez certains représentants de l'illuminisme et du romantisme français (Arthur Mc Calla) et chez Madame Blavatsky, Rudolf Steiner et René Guénon (Garry W. Trompf). 